\title{
Structure and dynamics of round turbulent jets ${ }^{a)}$
}

\author{
Paul E. Dimotakis, Richard C. Miake-Lye, ${ }^{\text {b) }}$ and Dimitris A. Papantoniou \\ California Institute of Technology, Pasadena, California 91125
}

(Received 2 December 1982; accepted 18 July 1983)

\begin{abstract}
Laser-induced fluorescence and particle streak velocity measurements were conducted to investigate the structure and dynamics of round turbulent jets. The results suggest that the farfield region of the jet is dominated by large-scale vortical structures, which appear to be axisymmetric or helical a large part of the time. Entrainment and mixing of the reservoir fluid with the jet fluid is found to be intimately connected with the kinematics of these structures. Unmixed reservoir fluid is found to reach and cross the jet axis.
\end{abstract}

\section{INTRODUCTION}

It has been known for some time that the near wake of a circular cylinder is organized in the form of a staggered array of vortices of alternating circulation to Reynolds numbers that are as large as have been investigated (Roshkol). The cylinder wake, however, had always been treated as a special case of high Reynolds number turbulent free shear flow, possessing a degree of organization considered atypical of such flows. This typical picture was challenged by the high Reynolds number plane shear layer investigations, initially by Brown and Roshko ${ }^{2,3}$ and subsequently by others, which revealed the existence of a large-scale organization of the flow, in the form of vortical structures with large vertical and spanwise coherence.

Evidence of large-scale organization in the transition (core) region $(x / d<5)$ of high Reynolds number axisymmetric jets can be found in the literature prior to the Brown and Roshko ${ }^{2,3}$ work (Brown, ${ }^{4}$ Bradshaw, Ferriss, and Johnson, ${ }^{5}$ Freymuth, ${ }^{6}$ Mollo-Christensen, ${ }^{7}$ and Crow and Champagne ${ }^{8}$ ). Subsequent work by Browand and Laufer ${ }^{9}$ and Yule, ${ }^{10}$ however, established rather unequivocally the existence and dominant role of these structures in this region of jet flow. Strong evidence can also be found that high Reynolds number turbulent boundary layers are also organized to a large extent, even though the corresponding structure and dynamics for that flow are not as well established at present. The review article by Cantwell ${ }^{11}$ discusses these issues and includes a useful list of references on large-scale structure organization of turbulent shear flows.

These findings are significant because they suggest that we may have to revise present concepts and models for turbulent transport mechanisms, which are based on turbulent fluxes proportional to local gradients of mean quantities, and form the basis of most theoretical and computational analyses of such flows at present. It should be borne in mind, as was pointed out by Corrsin and Kistler, ${ }^{12}$ that such models assume local fluctuations are of a scale much smaller than the transverse extent of the region of turbulent flow (see also Broadwell and Breidenthal ${ }^{13}$ ). There exists a strong motiva-

\footnotetext{
a) Invited lecture, XV International Symposium of Fluid Dynamics, Jachranka, Poland, 7-12 September 1981.

b) Present address: Stanford University, Stanford, California 94305.
}

tion, therefore, to investigate other flow geometries in an attempt to resolve whether these large structures are a universal property of turbulent shear flows, or a peculiarity of just a few. In the present paper we describe the results of one such investigation of the structure in the near and far field of round turbulent jets.

Flow visualization pictures of high Reynolds number turbulent jets (see Fig. 1, for example), in which dye or smoke has been used to render the "turbulence" visible, have traditionally suggested that it is possible to define an interfacial surface, which is topologically relatively simple, and which

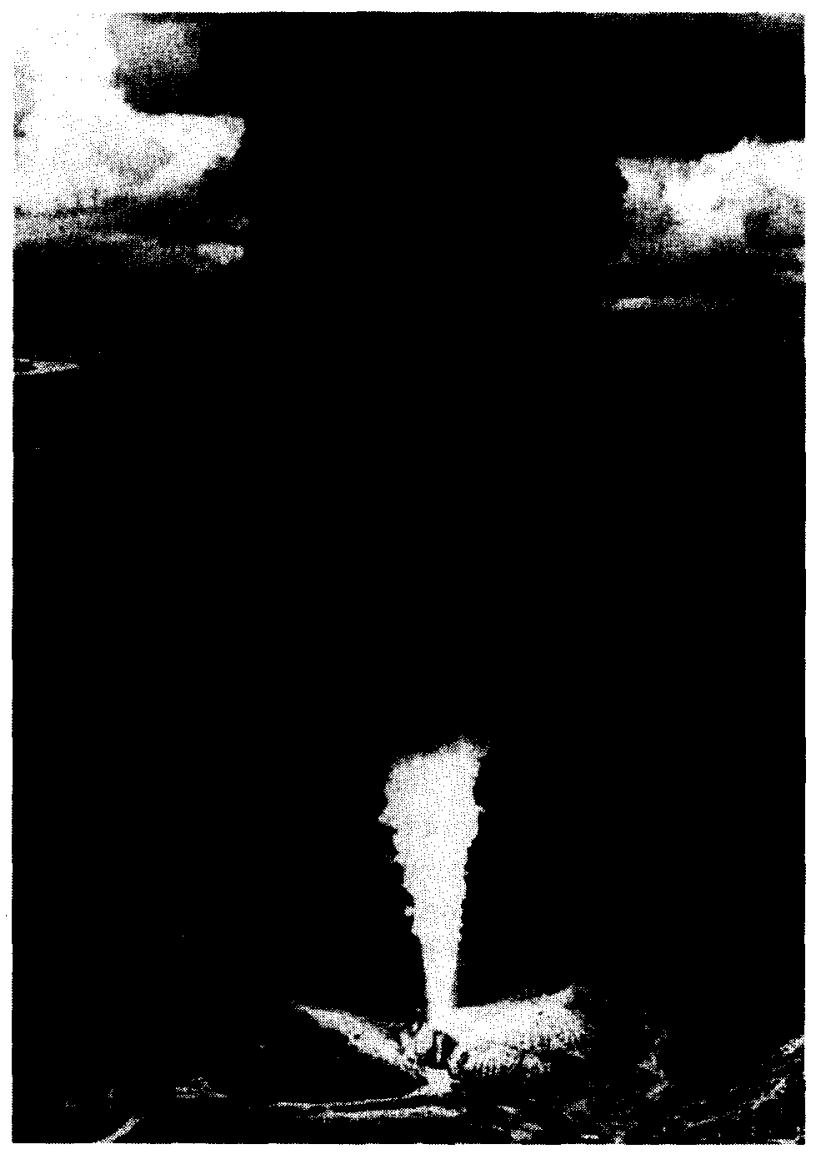

FIG. 1. High Reynolds number (three million pounds thrust) turbulent jet Rocket testing by Lockheed in the Los Angeles hills [from Las Angeles (Sunset-Lane, Menlo Park, CA, 1968), pp. 246-247]. 
separates the "turbulent" from the "nonturbulent" flow regions. The fact that this surface is not stationary has, in turn, suggested the concept of intermittency, defined as the fraction of the time that a particular point is inside the turbulent region (Corrsin and Kistler, ${ }^{12}$ see also Fig. 4.1 and related discussions in Tennekes and Lumley ${ }^{14}$ ). Consistent with this picture, entrainment of irrotational flow into the turbulence is discussed in terms of the diffuse, random walk propagation of the turbulent nonturbulent interface into the irrotational fluid, via the viscous laminar superlayer (Corrsin and Kistler, ${ }^{12}$ see also Townsend ${ }^{15}$ ).

It is important to realize, however, that conventional flow visualization images derived from such flows allow only an external (or integrated) view of the region of turbulence and are not generally capable of revealing the internal structure. A second shortcoming of conventional flow visualization techniques, especially at higher Reynolds numbers, is the problem of resolving the smallest viscous and mass diffusion scales of turbulence which decrease in size nearly inversely as the Reynolds number relative to the local transverse extent of the turbulent region. This lack of resolution, more often the case than not for both flow visualization and point measuring techniques, has also led to a picture of a turbulent region that is relatively homogenous and isotropic in its structure and composition.

To address these questions, new experimental techniques were utilized in the work reported here, which permitted an investigation of the interior of the transition and far field of a turbulent jet with a time and space resolution that was capable of resolving the Kolmogorov scale of the turbulence for a substantial fraction of the field of interest.

We would like to acknowledge that Liu et al. ${ }^{16}$ used laser fluorescence techniques to photograph the jet fluid concentration field on the plane of symmetry of the jet and to perform one- and two-point measurements of the jet fluid concentration fluctuations at a Reynolds number of 1600 . These experiments, however, which were not known to us at the time, do not appear to possess a spatial and temporal resolution that is commensurate with the small scale requirements corresponding to their jet operating conditions.

\section{EXPERIMENTAL APPARATUS AND MEASUREMENTS}

The present investigations were conducted in water, by discharging the jet through a shaped nozzle, from a plenum over a reservoir. The flow velocity was maintained nearly constant by driving the plenum with an air supply system metered through a micrometer-controlled variable throat sonic orifice valve, with a constant upstream pressure. See Figs. 2(a) and 2(b). Running times of 4-20 sec were sufficient to establish the flow and record the data in the form of still and motion picture photographs. The plenum and reservoir were quiesent before a solenoid valve, in series with the sonic orifice, was opened to initiate the flow. This arrangement obviates screens and flow-straightening devices, and yields a flow with negligible background turbulence levels.

Rhodamine $6 \mathrm{G}$ or sodium fluorescein was premixed in small concentrations with the jet fluid and an argon ion laser

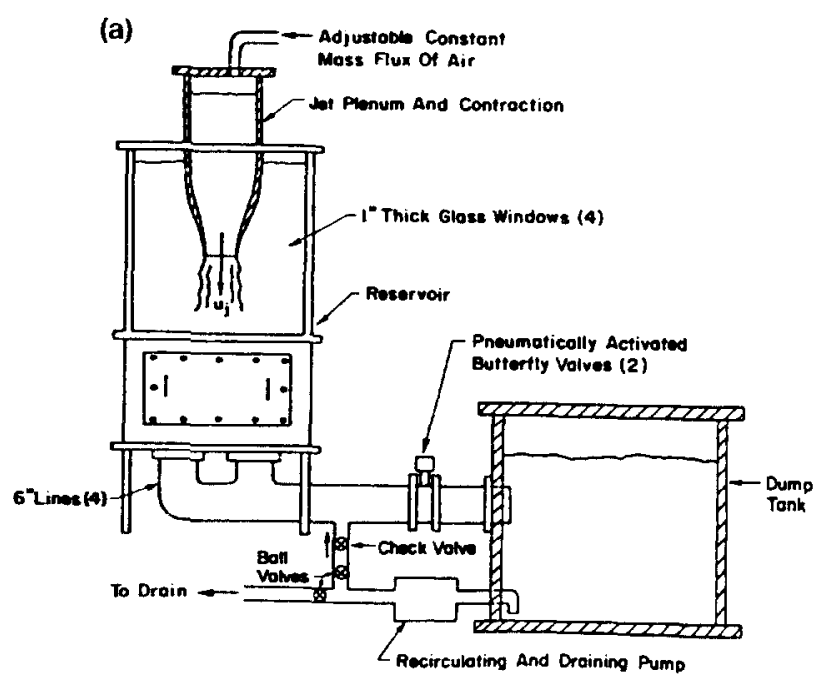

(b)

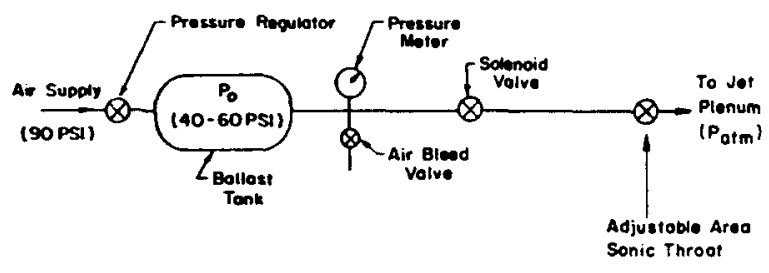

FIG. 2. Jet flow apparatus. (a) Jet flow facility. (b) Air supply system.

sheet was introduced into the test section from below or the side to illuminate the plane of symmetry of the jet. These dyes fluoresce with very short response times when excited with argon ion laser radiation and for nominal laser power densities and dye concentrations are characterized with linear response proportional to the local laser power and dye concentration (Schaefer, ${ }^{17}$ Dewey, ${ }^{18}$ Liu et al. ${ }^{16}$ ).

Using a combination of a long-focal-length spherical lens and a short-focal-length cylindrical lens, it was posssible to generate a laser sheet, focused to a thickness of 100-200 $\mu \mathrm{m}$ in the region of interest, with a sufficient width to cover the extent of the jet. Alternatively, the long-focal-length spherical lens could be used alone to collimate the laser beam along the jet axis.

The data were recorded either photographically, using the laser sheet configuration, by means of short-time-exposure still photographs, using a $35 \mathrm{~mm}$ camera with a $50 \mathrm{~mm}$ $f / 1.4$ lens, or with a $16 \mathrm{~mm}$ pin register motion picture camera at framing rates up to a few hundred frames per second (see Fig. 3), or using a collimated laser beam coincident with the jet axis, imaged on a self-scanning (RETICON) array of 1024 detectors along a line, spaced $25 \mu \mathrm{m}$ apart (see Fig. 4). The output of this array was then used to modulate an oscilloscope beam driven synchronously in a raster scan fashion to produce an $x-t$ diagram.

Under the operating conditions of the experiments reported here, the fluorescence intensity was proportional to the local laser intensity (which could be measured independently) and the instantaneous concentration of the fluores- 


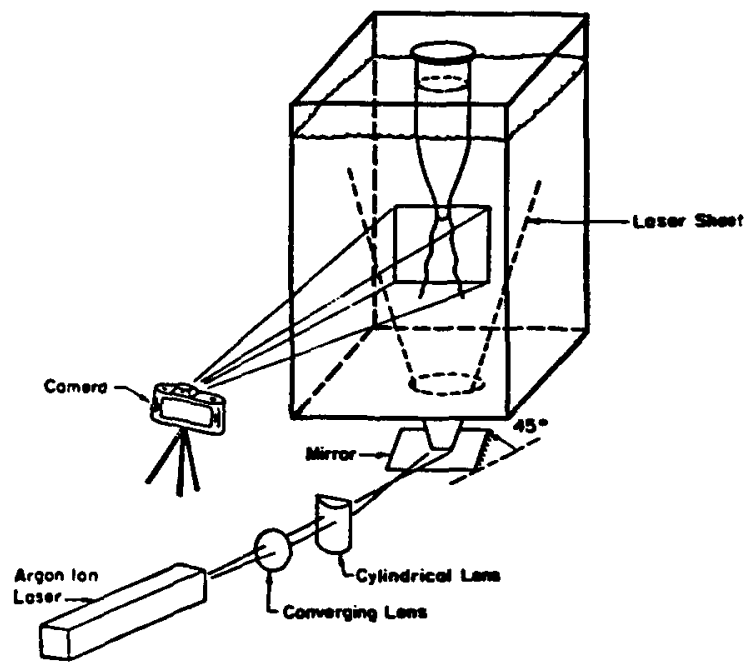

FIG. 3. Laser-induced fluorescence optics. Sheet illumination.

cent dye. Monitoring the resulting fluorescence allows a quantitative measurement of the concentration of jet fluid in the field of illumination, with a spatial resolution that exceeded that of conventional measurement techniques, and a time resolution that was adequate for the purposes of the present investigation.

It should be noted that the resulting fluorescence is emitted at a longer wavelength at which the dye is characterized by a negligible absorption cross section. Consequently, the medium is always in the optically thin regime as far as the fluorescence emission is concerned. In this manner, a slice through the turbulence is possible, circumventing the problems of conventional flow visualization techniques, which are limited to an external (or integrated) view of the turbulent region.

\section{RESULTS AND DISCUSSION}

Figures 5(a) and 5(b) show still photographs of the concentration of jet fluid in the plane of symmetry of a jet at a Reynolds number of approximately 2500 . The initial transition region is seen to end with the amplification and rollup of an axisymmetric disturbance, followed by the end of the core region and finally by the linearly expanding turbulent region through the end of the picture. Several important conclusions can be drawn from these pictures and numerous others like them.

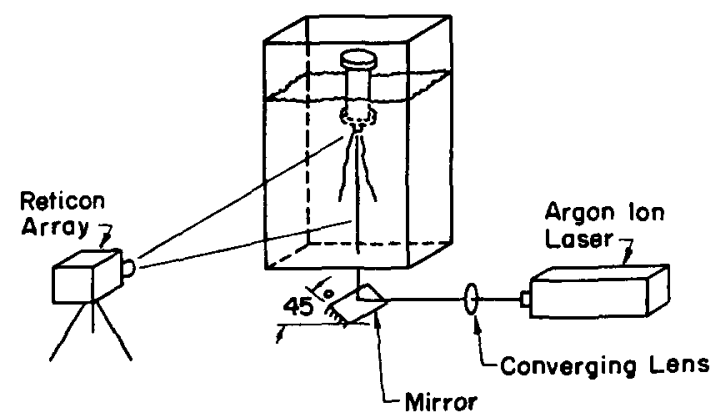

FIG. 4. Laser-induced fluorescence optics. Line illumination along the jet axis. (a)

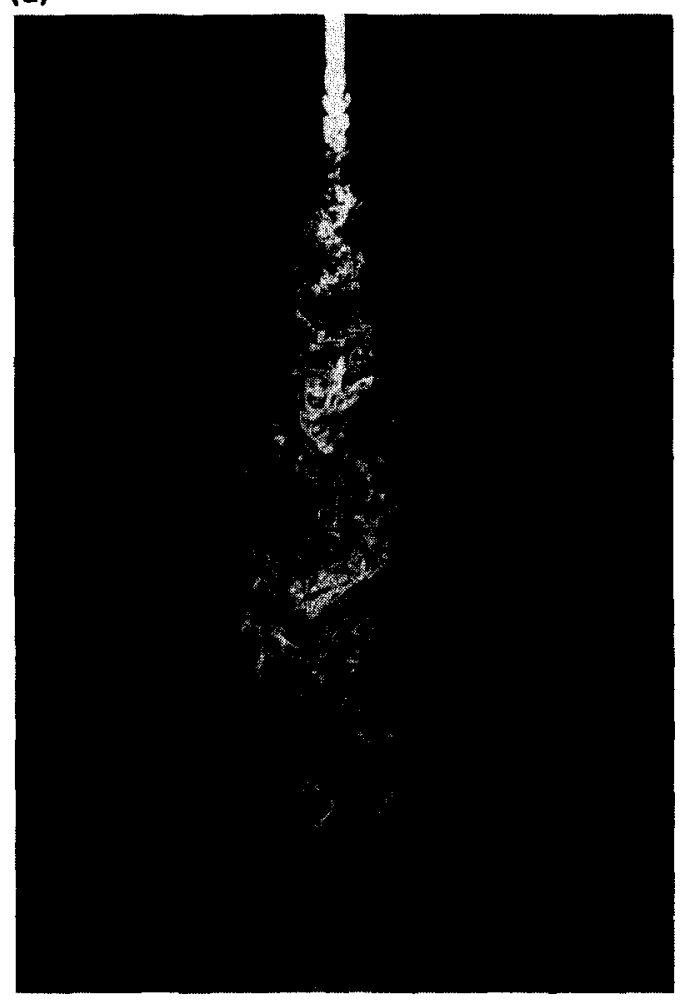

(b)

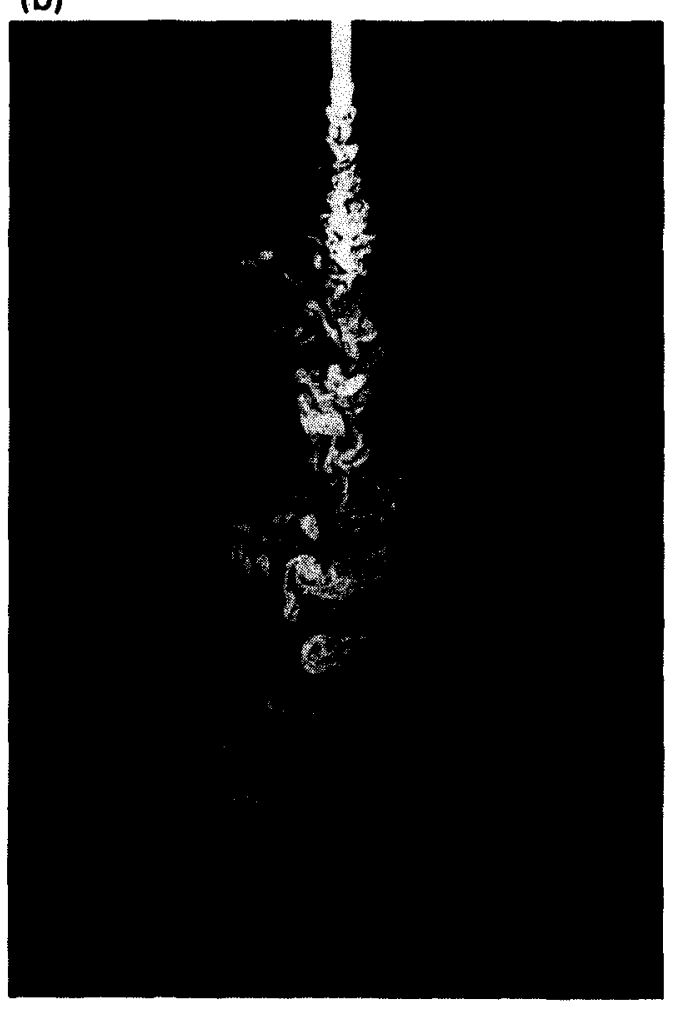

FIG. 5. Jet fluid field in the plane of symmetry of the jet $(\operatorname{Re}=2500$, $d=0.75 \mathrm{~cm}, 0<x / d<35$ ). (a) Typical structure. (b) Evidence of spiral mode. 
The notion of a topologically simple interface separating the turbulent from the nonturbulent flow is seen to be questionable on the basis of this view of the jet. Even if such an interfacial surface could be defined, it would appear to possess all the scales of the turbulent spectrum. The concept of being "inside the turbulence," as opposed to "outside the turbulence," on which the notion of intermittency is based, is highly questionable in this light. Perhaps the concept of a fractal surface (Mandelbrot ${ }^{19}$ ) might be more apt as a description of the turbulent-nonturbulent interface.

If we were to ignore this difficulty, and accept, as is often done, an operational definition of intermittency as the presence of jet fluid, we would be forced to conclude from these pictures that we must assign an intermittency factor that is less than unity on the axis of the jet; unmixed reservoir fluid can be seen to reach and even cross the jet axis. Perhaps a more appropriate definition of intermittency might be in terms of fluctuating vorticity, and vorticity fluctuations diffuse with the kinematic viscosity. Mixing of the reservoir fluid with jet fluid on small scales, on the other hand, occurs via the coefficient of mass diffusion; and, for water, the ratio of the two molecular diffusion rate coefficients (Schmidt number) is approximately $10^{3}$ (of order unity for gases). Nevertheless, and until adequate time- and space-resolved vorticity measuring techniques are developed, we can say on the basis of the available data that since the unmixed fluid appears to be associated with scales of all sizes, it would seem unlikely that changing from a liquid to a gas would alter these conclusions in a qualitative manner.

It should be noted that, in the data of Figs. $5(\mathrm{a})$ and $5(\mathrm{~b})$, the resolution in the downstream half of the picture matches or exceeds the Kolmogorov scale in all three dimensions. A reduction in resolution, which was easily achieved with no other change in our experiments by removing the spherical collimating lens, resulted in pictures more closely resembling conventional flow visualization pictures of high Reynolds number jets.

Another issue that must be readdressed, in view of these observations, is entrainment. If the regions of turbulence cannot be circumscribed in the conceptual manner that had been assumed to be valid in the past, we must revise our notion of entrainment. It cannot as readily be identified with the propagation of the turbulent-nonturbulent "superlayer" interfacial surface of Corrsin and Kistler into the irrotational fluid. To investigate this issue further, particle streak velocity field slices (see also Dimotakis et al ${ }^{20}$ ) in the plane of symmetry of the same jet at the same conditions were recorded. An example of such data is depicted in Fig. 6. This picture and many others, as well as motion picture data which monitor the entrainment process with the aid of a combination of laser-induced fluorescence labeling of the jet fluid and neutrally bouyant scattering particles in the reservoir fluid, suggest that the fluid we would associate with the irrotational region in the periphery of the jet is set in motion through the induced velocity field of the vortical structures in the jet. Fluid at fairly large distances from the turbulence (here defined as the region of fluctuating vorticity) is "committed" to enter the turbulent region. The entrainment mechanism appears to be best described as kinematic, a con-

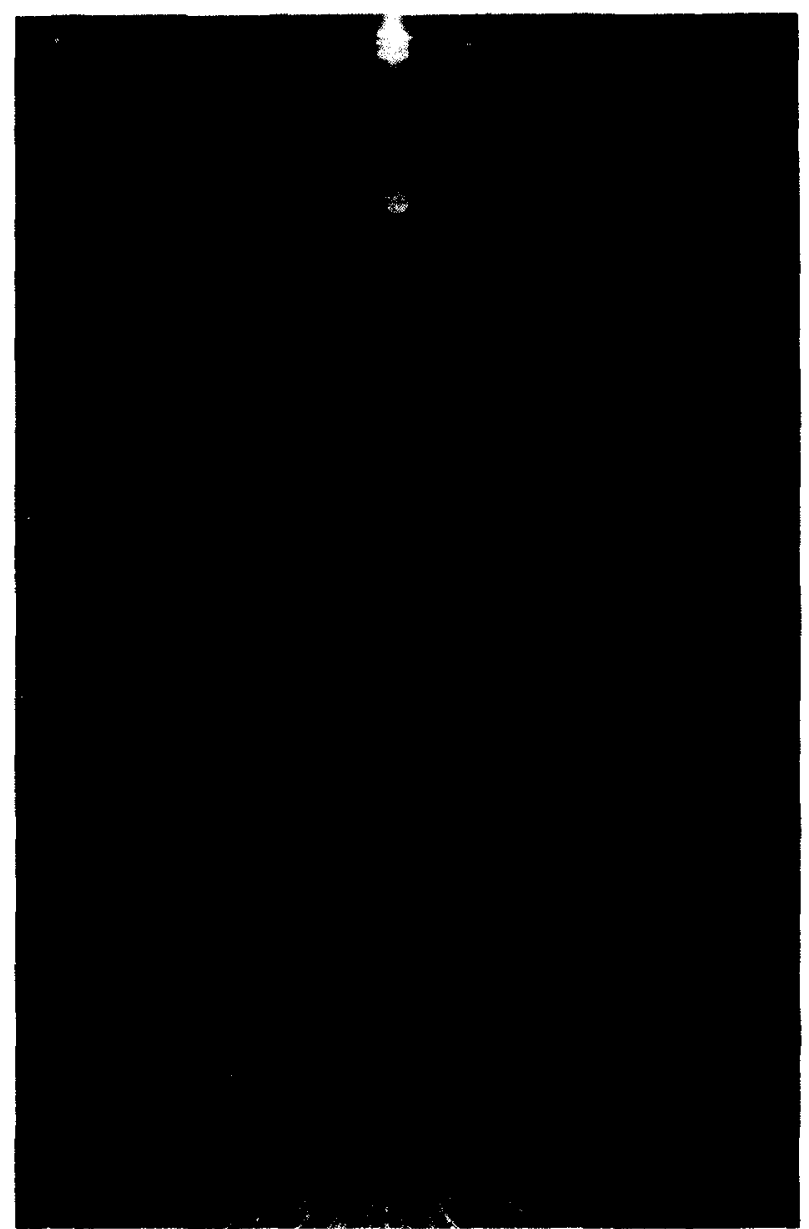

FIG. 6. Particle streak velocity field in the plane of symmetry of the jet. Jet and flow conditions as in Figs. 5(a) and 5(b).

sequence of Biot-Savart induced velocities at some distance from the turbulence proper, rather than diffusive. This is also consistent with the deep ingestations of pure reservoir fluid that are evident in the photographs of Figs. 5(a) and $5(\mathrm{~b})$.

In the photograph of Fig. 5(b), the jet fluid appears to be associated with a large-scale motion whose intersection with the plane of symmetry is in the form of a zigzag pattern of increasing period and amplitude. This would be consistent with assuming that the jet three-dimensional structure, at the instant of this particular photograph, was in the form of an expanding spiral (please note that the jet has no swirl). While this is not always the case [e.g., Fig. 5(a)], we can say that, based on a substantial amount of data derived from both still and motion picture photography, that the far field of the jet appears to be organized in the form of large-scale concentrations of vorticity which are approximately axisymmetric, or spiral, or in a transitional state inbetween these two configurations. This is illustrated in the sequence depicted in Fig. 7, obtained by printing every other frame from a film recorded using the $35 \mathrm{~mm}$ camera driven by a motor at 3.5 frames/sec. The jet Reynolds number for this sequence was 650. Approximately 160 jet diameters are included in each frame. Note that the jet, which is not in any obviously organized state at the beginning of the sequence, has formed 

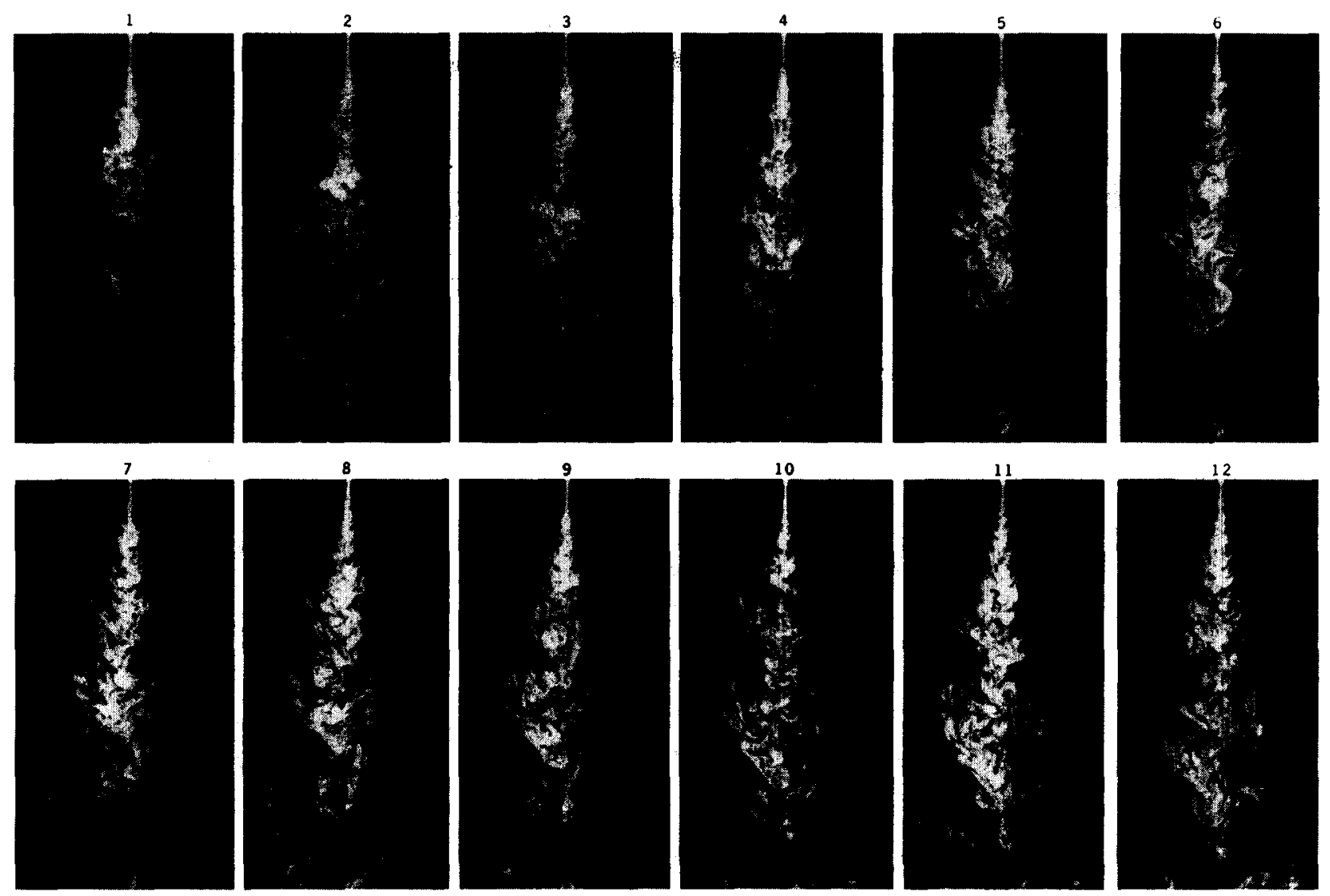

FIG. 7. Creation of an axisymmetric structure at 160 diameters $(1.7$ frames $/ \mathrm{sec}$ sequence, $\operatorname{Re} \approx 650$, and $d=0.25 \mathrm{~cm})$.

a well-defined axisymmetric structure at the downstream end of the frame, by the end of the sequence.

This sequence was recorded a short time after Hans Liepmann suggested the following general argument (private communication). If we were to assume that, at some instant, turbulent shear flow were to find itself in a disorganized state described by a stochastic fluctuation field of small incoherent eddies, then certainly at that time, and for a short while thereafter, the notions of gradient transport and eddy diffusivity should be valid. The region undergoing turbulent shear would then be found unstable to the formation of an organized structure, whose scale would span the transverse extent of the sheared region. In the case of the jet, such a structure would correspond to the axisymmetric $(m=0)$ and helical $(m=1)$ modes predicted by linearized stability analysis (Michalke ${ }^{21}$ ).

To study the dynamics of these structures, a different set of measurements was performed. Using the laser as a collimated beam coincident with the jet axis, the fluorescence was imaged on a 1024 array of detectors, as described in Sec. II. The resulting data correspond to an $(x, t)$ diagram of the intersection of jet fluid with the illuminated jet axis. An example of such data is depicted in Fig. 8. Approximately 33 jet diameters are included in the horizontal extent of the picture, which were scanned for $10 \mathrm{sec}$. The jet Reynolds number for this recording was approximately 500 . The detector array signal contrast was enhanced electronically and also inverted, so that the white streaks in this picture corre- spond to reservoir fluid (no fluorescence). The jet exit can be detected at the left of the picture from the small amount of light scattered by the mouth of the jet nozzle.

Several observations are suggested by the data in Fig. 8 and other similar recordings. It can be seen that no reservoir fluid can be detected for the first six to seven diameters downstream of the jet exit. This is consistent with the extent of the core region, which in this case is extended by a transition region of a few diameters due to the low value of the Reynolds number. See also the initial region in Figs. 5(a) and 5 (b). Streaks of reservior fluid can be seen to convect downstream with celerities which appear piecewise nearly constant. Jet fluid elements can be seen to run into each other, the upstream element overtaking and coalescing with the downstream one. Note that this might have been anticipated, since an upstream structure will typically be propagating faster than its downstream neighbor. Celerities appear to decrease rather abruptly with each such coalescence. The average celerity, at each streamwise value of the coordinate, decreases smoothly and monotonically only in an ensemble average sense. Streaks can be seen to propagate with no interactions and with nearly constant celerity for a number of diameters which can be seen to be as large as one-half the value of the local $x / d$.

To investigate further the mechanisms of entrainment and subsequent mixing of the reservoir fluid with the original jet fluid, the small jet that was used in the sequence of Fig. 7 was also operated at a Reynolds number of approxi- 


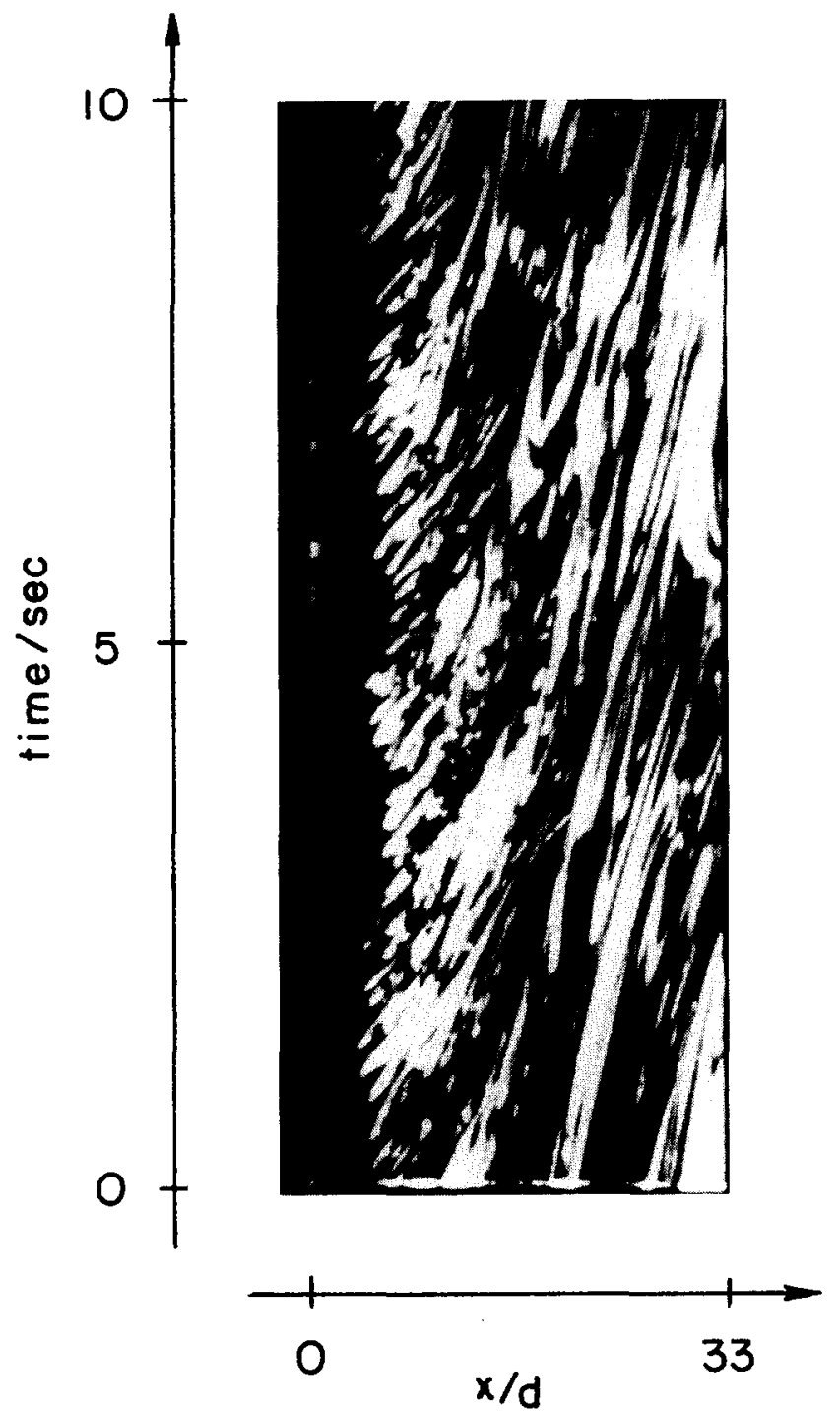

FIG. 8. Concentration of reservoir fluid along the jet axis, as a function of time $(\operatorname{Re} \approx 500$ and $d=0.75 \mathrm{~cm})$.

mately 10000 . Typical photographic data at these conditions are depicted in Fig. 9. It should be noted that at this Reynolds number, the sheet thickness is not sufficient to resolve the smallest scales, and serves to indicate the local (spatial) mean of the concentration of jet fluid. It should also be noted that the time exposure was not sufficiently short to freeze the motion of the fluorescent jet fluid in the region near the jet exit (small $x / d$ ), where the flow velocity is still high. The sharp fronts of monotonically decreasing concentration levels are noteworthy. They also suggest, even within the region of turbulent shear flow, that the mixing is not dominated by a gradient transport mechanism but rather intimately connected with the motion and dynamics of the large scales of vorticity in the flow in a manner reminiscent of two-dimensional shear layers.

\section{THE TRANSITION REGION}

Our data, especially at the higher Reynolds numbers using the larger diameter jet nozzles of 2.5 and $7.5 \mathrm{~cm}(1.0$ and $3.0 \mathrm{in}$., respectively), confirm the existence of the highly organized large-scale structures in the transition (core) re-

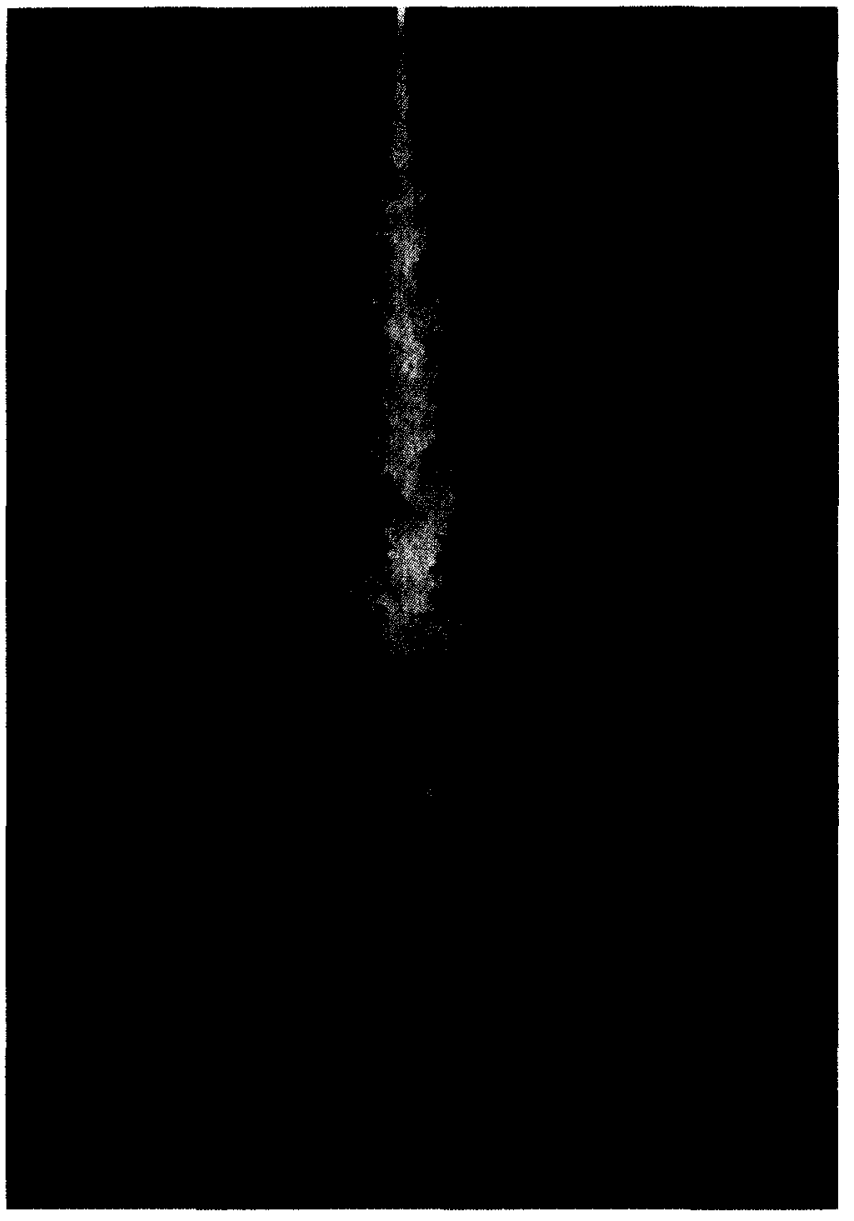

FIG. 9. Concentration of jet fluid in the plane of symmetry of the jet $(\operatorname{Re} \approx 10000, d=0.25 \mathrm{~cm}$, and $0<x / d<200)$.

gion of the jet $(0<x / d<4-5)$ that have been documented previously. ${ }^{4-10}$ We find, however, that for our larger nozzles operated at the higher Reynolds numbers, in which the momentum thickness of the boundary layer at the exit plane of the jet is several hundred times smaller than the jet radius, that the dominant mode after one or two jet diameters is not axisymmetric $(m=0)$, but rather helical $(m=1)$.

It is interesting to note that these observations are in qualitative agreement with the linear stability analysis predictions of Michalke, ${ }^{22}$ who finds that the axisymmetric $(m=0)$ mode is the most unstable, if the shear layer momentum thickness is small compared to the jet radius, but that for a thicker shear layer, corresponding to locations further downstream in the jet core region, the spiral $(m=1)$ mode is the most unstable. Again, as with the apparent success of linear stability analysis of the full jet velocity profile that would apply to the far field, it is not clear why linear analysis should be relevant; the velocity deviations from a mean profile are not small by any measure.

The large-scale helical vortical structures appear to organize into left- or right-handed spirals, which are capable of undergoing coalescence interactions, similar to the ones observed for their axisymmetric (as well as their plane shear layer) counterparts. This is evident in our motion picture data and consistent with the experimental observations of 
Browand and Laufer, ${ }^{9}$ who estimated that the spiral mode was dominant approximately $50 \%$ of the time, at Reynolds numbers comparable to the values in our present investigations. The jet in this region, as well as in the far field, appears capable of switching from an axisymmetric to a spiral mode and back again, in a way that is not clear at present. Our observations suggest that the resulting structure is much like a bedspring which starts as a nearly closed loop, continues as a spiral, and closes again as a loop. It goes without saying, that additional lines of vorticity must also exist connecting the top loop with the bottom loop, in deference to the vorticity theorems.

\section{REYNOLDS NUMBER EFFECTS}

One of the difficult questions to answer in any investigation of fully developed turbulent flow is the dependence of the various phenomena and observations on the Reynolds number. One would like to know, for example, whether a particular feature of the flow, observed in the low to moderate Reynolds numbers usually achievable in a conventional laboratory environment, can be assumed to exist in the limit of high Reynolds numbers. This has been the topic of an active discussion in the last few years, following the realization of the significance of the large-scale structures in turbulent shear flow.

In our view, the evidence that the large-scale structures found to be associated with turbulent free shear flows persist to the highest Reynolds numbers is quite compelling. In the case of the shear layer, this question was specifically addressed by Dimotakis and Brown, ${ }^{23}$ who showed by means of flow visualization techniques that the large-scale structures persist to Reynolds numbers of the order of $10^{7}$. In the case of wakes, the similarity in the photograph in Fig. 16 of the review article by Cantwell, ${ }^{11}$ between the wake of Argo Merchant oil spill at Nantucket $\left(\operatorname{Re} \approx 10^{8}\right)$ and the small water channel wake behind an inclined plane $\left(\operatorname{Re} \approx 10^{3}\right)$, is noteworthy. The general argument to be made here is that (i), in the absence of the effects of neighboring solid boundaries and at sufficiently high Reynolds numbers, viscosity is not important in determining the dynamics of the large scales of turbulence in free turbulent flows; and (ii), to the extent that the large scales in the flow dominate the aspects of interest of the flow, the Reynolds number would not be a parameter of the problem.

The value of the Reynolds number is of course important in determining the size of the smallest scales in the turbulent spectrum of fluctuations. By corollary, it is also important in determining the temporal and spatial resolution specifications of any measurements designed to address issues such as intermittency and mixing related to the smallest scales of turbulence.

The Reynolds number is also important in defining, for a given flow geometry, the size of the initial momentum thickness of the velocity profile at the exit plane of the jet. This in turn will set the effective jet diameter as well as the virtual origin for the far-field self-preserving region. To the extent, however, that the aspects of interest of the flow are unrelated to these considerations, then Reynolds number, provided it is in excess of some minimum value, should not be an important parameter.

This hypothesis is supported by the chemically reacting jet data of Hottel, ${ }^{24}$ who found that the distance required to react all of the jet fluid reached its asymptotic value at a Reynolds number of approximately $2500-3000$. Similar evidence can be found in the work of Shea, ${ }^{25}$ who detected no difference in the behavior of his reacting jets between his lowest Reynolds number of 4000 and the highest of 32000 . It should be noted, however, that these results are badly at variance with the measurements of Ricou and Spalding, ${ }^{26}$ who found that jet entrainment did not reach an asymptotic value until a Reynolds number of 40000 . The reasons for this discrepancy are not clear as of this writing. It is our feeling, however, that Hottel's data, which measure the end result of the whole entrainment and molecular mixing process, provide the definitive evidence on this issue. Moreover, as that data was for a jet in water, the estimate of the minimum Reynolds number for the asymptotic behavior should be considered as an upper bound for a gas jet (Schmidt number $\approx 1$ ).

\section{CONCLUSIONS}

We believe we have evidence to suggest that large-scale vortical structures dominate the dynamics of fully developed turbulent jets, not only in the transition region, as has been documented previously, but also in the far field. The entrainment and subsequent mixing of the ambient reservoir fluid, into which the jet is discharging, is intimately associated with the kinematics of these structures and, in particular, does not appear appropriately describable as a gradient diffusion phenomenon. Our observations also suggest that the notion of intermittency may have to be modified and that serious operational difficulties may exist in attempting intermittency measurements. The large-scale structures are found to be either nearly axisymmetric, or spiral, or in a transitional state between these two configurations.

These results have important implications in applications of chemically reacting and combusting jets, as they suggest that the mixing environment dictated by the fluid mechanics may be quite different than the one suggested by classical turbulence theories.

\section{ACKNOWLEDGMENTS}

This work was supported by the Air Force Office of Scientific Research through Contracts No. F44620-76-0046 and No. F49620-79-C-0159, and by the Boeing Commercial Airplane Company, Contract No. Y-481468-0935N.

\footnotetext{
'A. Roshko, J. Fluid Mech. 10, 345 (1961).

${ }^{2}$ G. L. Brown and A. Roshko, AGARD-CP-93, Paper No 23, 1971.

${ }^{3}$ G. L. Brown and A. Roshko, J. Fluid Mech. 64, 775 (1974).

${ }^{4}$ G. B. Brown, Proc. Phys. Soc. 47, 703 (1935).

${ }^{5}$ P. Bradshaw, D. H. Ferriss, and R. F. Johnson, J. Fluid Mech. 19, 591 (1964).

${ }^{6} \mathrm{P}$. Freymuth, J. Fluid Mech. 25, 683 (1966)

${ }^{7}$ E. Mollo-Christensen, J. Appl. Mech. 34, 1 (1967).
} 
${ }^{\text {k}}$ S. C. Crow and F. H. Champagne, J. Fluid Mech. 48, 547 (1971).

${ }^{9} \mathrm{~F}$. K. Browand and J. Laufer, in Fourth Symposium on Turbulence in Liquids (University of Missouri-Rolla, 1975, edited by J. L. Zakin and G. K. Patterson (Science, Princeton, NJ, 1977).

${ }^{16}$ A. J. Yule, J. Fluid Mech. 89, 413 (1978).

${ }^{11}$ B. J. Cantwell, Ann. Rev. Fluid Mech. 13, 457 (1981).

${ }^{12}$ S. Corrsin and A. L. Kistler, NACA Report R-1244, 1955.

${ }^{13}$ J. E. Broadwell and R. E. Breidenthal, J. Fluid Mech. 125, 397 (1982).

${ }^{14} \mathrm{H}$. Tennekes and J. L. Lumley, A First Course in Turbulence (MIT, Cambridge, MA, 1972).

${ }^{15}$ A. A. Townsend, The Structure of Turbulent Shear Flow (Cambridge U.P., Cambridge, 1976), 2nd ed.

${ }^{16}$ H. -T. Liu, J.-T. Lin, D. P. Delisi, and F. A. Robben, NBS Special Publication 484, Proceedings of the Symposium on Flow in Open Channels and Closed Conduits held at NBS, Gaithersburg, Maryland, 23-25 February
1977.

${ }^{1}$ F. P. Schaefer, Dye Lasers (Springer, Berlin, 1973), Vol. 1.

${ }^{14}$ F. C. Dewey Jr., AGARD-CP-193, Paper No. 17, 1976.

${ }^{19}$ B. B. Mandelbrot, Fractals. Form, Chance and Dimension (Freeman, San Francisco, 1977).

${ }^{21}$ P. E. Dimotakis, F. D. Debussy, and M. M. Koochesfahani, Phys. Fluids 24, 995 (1981)

"A. Michalke, Z. Fluqwiss. 19, 319 (1971), translated into English, NASATM-75190, 1977.

2:A. Michalke and G. Herman, J. Fluid Mech. 114, 343 (1982)

${ }_{23}^{23}$. E. Dimotakis and G. L. Brown, J. Fluid Mech. 78, 535 (1976)

${ }^{24} \mathrm{H}$. C. Hottel, 4th (International) Symposium on Combustion (Williams and Wilkins, Baltimore, MD, 1953), p. 97.

${ }^{25}$ J. R. Shea, J. Fluid Mech. 81, 317 (1977).

${ }^{2 h}$ F. P. Ricou and D. B. Spalding, J. Fluid Mech. 11, 21 (1961). 